\title{
INFLUÊNCIA DA BANDAGEM NEUROMUSCULAR NO DESEMPENHO DO SALTO VERTICAL
}

\author{
Matteus Lisandro Arêas Tinoco \\ Graduado em Fisioterapia/ISECENSA/RJ \\ felipsjorge@gmail.com
}

Thiago Geovanini de Oliveira Tavares

Graduado em Fisioterapia/ISECENSA/RJ

thiago07_got_@hotmail.com

\section{Anderson Pontes Morales}

Mestre em Ciências da Motricidade Humana/UCB/RJ

andersonmrl@hotmail.com

\section{Felipe Sampaio Jorge}

Mestre em Engenharia Biomédica/UNIVAP/SP

felipsjorge@gmail.com

Recebido: 15 de abril de 2012. Revisado: 21 de maio de 2012. Aceito: 31 de julho de 2012. Publicado online: 27 de outubro de 2012.

\section{RESUMO}

Diversas modalidades esportivas utilizam o salto vertical como um dos principais movimentos. Com a maior exigência do desempenho físico no meio esportivo, se faz necessário intervenções que venham a otimizar o desempenho dos indivíduos. A Bandagem Neuromuscular consiste em uma fita adesiva que pode ser esticada em até $140 \%$ do seu tamanho original aderindo facilmente à pele, tendo como objetivo regular tônus muscular, aliviar dor, correção articular. É frequentemente utilizada no âmbito esportivo com o intuito de otimizar o desempenho dos atletas, embora sem evidências científicas que comprovem sua eficácia. Este estudo propõe-se a verificar se a Bandagem Neuromuscular aplicada nos músculos quadríceps e tríceps sural promove a melhora do desempenho do salto vertical. Foi realizado um ensaio clínico cruzado randomizado, com 12 voluntários sedentários, divididos em 2 grupos: grupo controle(CNTRL- sem bandagem) e grupo com bandagem (CB), onde eram submetidos a uma série de saltos sobre uma plataforma específica para a análise das variáveis (número de saltos, altura, tempo de solo, tempo de voo, potência relativa, potência absoluta) necessárias. As avaliações foram realizadas nos momentos pré- aplicação, pós 10 minutos, pós 24 horas e pós 48 horas. Não houve diferença estatisticamente significativa $(p>0,05)$ no desempenho do salto vertical, quando comparados os grupos com bandagem e sem bandagem. A aplicação da Bandagem Neuromuscular não interferiu na performance muscular e na resistência a fadiga, mesmo após 48 horas de aplicação.

Palavras chave: Bandagem Neuromuscular, desempenho, salto vertical, fadiga. 
ABSTRACT

Many sports uses the vertical jump as one of the most essential motion. With the higher request of the physical performance at sports, It's necessary interventions able to optimize the performance of individuals. The neuromuscular Bandage consists of a tape that can be stretched up to $140 \%$ of its original size easily adhering to the skin, aiming to regulate muscle tone, relive pain, joints correction. It's often used in sports in order to optimize athletes performance, although don't exists anys cientific evidence to prove their efficacy. This study proposes to verify if the Neuromuscular Bandage applied at the muscles quadriceps and triceps surae promotes the improvement of vertical jump performance. We performed a randomized crossover trial, with 12 sedentary volunteers that were divided into 2 groups: control group (CNTRL- without bandage) and banded group $(\mathrm{CB})$, where they were subjected to a series of jumps on a specific platform for analysis of the variables (number of jumps, height, ground time, flight time, relative power, absolute power) required. The evaluations were conducted in the moments pre application; after 10 minutes, after 24 and after 48 hours. No statistically significant difference $(p>0.05)$ in vertical jump performance when comparing the groups with and without bandage. The application of Neuromuscular bandage did not affect the performance or resistance to muscle fatigue, even after 48hof application.

Keywords: Neuromuscular Bandage, performance, vertical jump, fatigue.

\section{INTRODUÇÃO}

A busca aprofundada de conhecimento sobre a funcionalidade do corpo humano por parte dos profissionais da área da saúde e esportiva tem aumentado gradativamente de acordo com o aumento no número de indivíduos que praticam atividades esportivas, profissionalmente ou não (SILVA, MAGALHÃES, GARCIA, 2005).

Diversas modalidades esportivas exigem um alto desempenho físico e a potência, principalmente dos membros inferiores, é ainda mais (verificar a concordância) determinante em modalidades que apresentam o salto como um dos principais movimentos (FURTADO, de MELO, GARCIA, 2006). O salto vertical consiste em um complexo movimento que une várias ações motoras envolvendo membros inferiores e superiores simultaneamente, estando presente de forma significativa em diversas modalidades esportivas, como o futebol, voleibol, basquetebol, handebol (GARCÍA-LOPEZ et al, 2008).

A avaliação do salto vertical possibilita avaliar a força gerada pela musculatura de membros inferiores. A análise do salto vertical implica algumas variáveis que determinam a performance, são elas: velocidade de deslocamento, potência realizada contra o solo, altura do salto e tempo de voo. (SILVA, MAGALHÃES, GARCIA, 2005; PEREIRA et al, 2009). O desempenho que o indivíduo apresenta na execução do salto vertical está intimamente relacionado com a capacidade da força e potência realizada pela musculatura extensora dos membros inferiores (GARCÍA-LOPEZ et al, 2008). 
Devido a maior exigência do desempenho físico no âmbito esportivo, se faz necessário intervenções que venham a otimizar o desempenho dos indivíduos. A Bandagem Neuromuscular (BNM) é uma técnica relativamente nova utilizada no processo de reabilitação considerada hoje pelos fisioterapeutas como um método de manutenção e apoio na reabilitação modulando vários processos fisiológicos (SLUPIK et al, 2007; HALSETH et al, 2004). Criada por Kenzo Kase em 1996, a BNM constitui uma nova técnica de bandagem que tem característica elástica podendo ser estirada em até $140 \%$ do seu tamanho original fazendo com que a força de retração elástica da fita providencie também sobre a pele a mesma força de retração elástica (KASE et al, 2003).

A proposta da BNM é que, quando aplicada corretamente o seu uso promova mecanismos como: correção da função muscular fortalecendo músculos debilitados, melhora da circulação sanguínea e linfática, reduzindo o processo de inflamação na região afetada, diminuição da dor por supressão neurológica, e reposicionamento das articulações subluxadas aliviando a tensão e ajudando a devolver a função desejada da musculatura e da fáscia. (SIJMONSMA, 2007).

Outro mecanismo proposto, porém pouco conhecido é que pela sua aplicação ocorra um aumento na propriocepção por aumentar o estímulo em mecanoceptores cutâneos. Após a aplicação da BNM, esta produz estímulos somatosensoriais aferentes e estímulos mecânicos constantes que são percebidos a nível cortical produzindo uma resposta motora (SANTOS et al, 2010; SLUPIK et al, 2007).

Devido ao grande e crescente uso da BNM no âmbito esportivo, porém sem evidências científicas que sustentem sua influência sobre a performance, este estudo propõe-se a verificar se o uso da BNM interfere no desempenho do salto vertical através de dados captados por uma plataforma de salto.

\section{METODOLOGIA}

O estudo caracteriza-se como um ensaio clínico cruzado randomizado. A randomização foi realizada através de um sorteio para selecionar os 12 voluntários em dois grupos: Com Bandagem (CB - neste grupo foi realizada a aplicação da BNM com a técnica de ativação sobre os músculos quadríceps e tríceps sural) e Controle (CNTRL grupo sem a aplicação da BNM), sendo que cada voluntário participou de ambos os grupos.

Os voluntários tinham idade entre 18 e 30 anos, altura entre 1,60 e 1,90 cm e massa corporal entre 58 a $100 \mathrm{~kg}$. O critério de exclusão determinou que indivíduos com lesão articular ou musculoesquelética que envolvem a mecânica do salto ou com hipersensibilidade da pele também puderam participar do estudo. Todos os voluntários assinaram o Termo de Consentimento Livre e Esclarecido, sendo o estudo aprovado pelo Comitê de Ética em Pesquisa (CEP) do ISECENSA, segundo padrões para pesquisa em seres humanos. A análise dos saltos foi realizada no laboratório de análise e movimento, situado na Clínica Escola Maria Auxiliadora.

Primeiramente, foram colhidos os seguintes dados: nome, idade, altura e massa corporal total. No grupo $\mathrm{CB}$ os voluntários realizaram tricotomia e limpeza com álcool $70 \%$ da região anterior da coxa e posterior de perna para a aplicação da BNM.

Para a ativação do músculo quadríceps (Figura 1), o indivíduo se encontrava

Persp. online: biol. \& saúde, Campos dos Goytacazes, 7 (2), 62-75, 2012 
em decúbito dorsal. Foi colado a base da BNM um pouco abaixo da espinha ilíaca antero- inferior, sem tensão. Após foi solicitado uma leve flexão de quadril e extensão total de joelho sendo colada a BNM com tensão de $20 \%$, em forma de "Y" sobre o ventre muscular do músculo quadríceps, em direção a sua inserção distal (tendão patelar e a tuberosidade da tíbia).

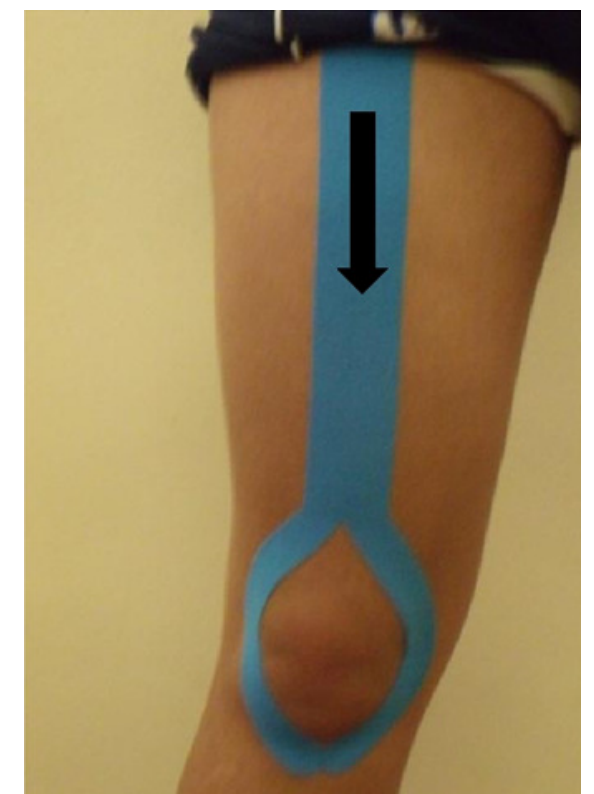

Figura 1: Ativação do músculo quadríceps. Técnica em "Y"aplicada da região abaixo da espinha ilíaca antero- superior até o tendão patelar com tensão da fita de cima para baixo de $20 \%$.

Já na ativação do tríceps sural (Figura 2) foi solicitado que o indivíduo ficasse em pé, sendo colada à base da BNM sobre a região poplítea com o músculo em repouso, sem tensão. Em seguida, o indivíduo ficou na ponta dos pés para que fosse promovido o encurtamento do tríceps sural e colado a BNM com $20 \%$ de tensão, em forma de "I" sobre o ventre muscular em direção ao tendão de Aquiles. 


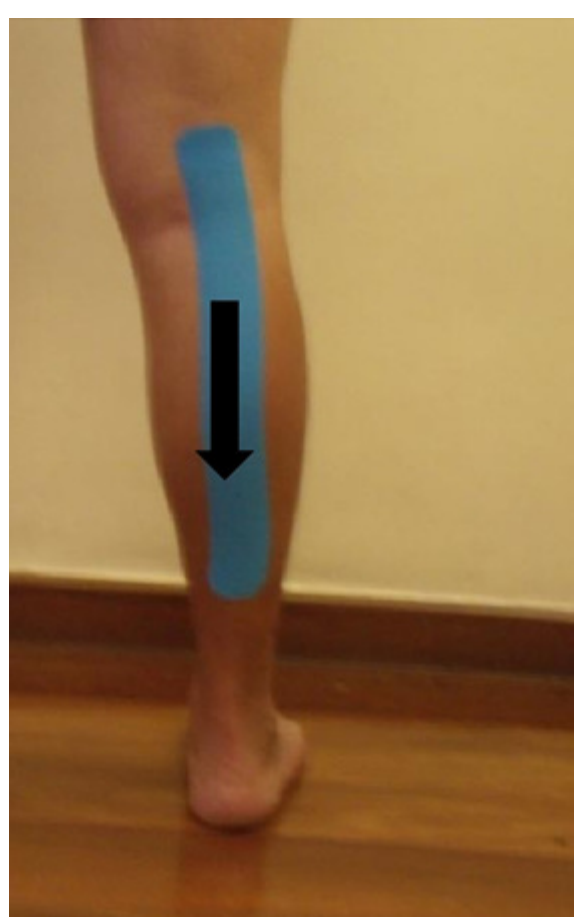

Figura 2: Ativação do músculo tríceps sural. Técnica em "I" aplicada da região poplítea até o tendão de Aquiles com tensão da fita de cima para baixo de $20 \%$

Para coleta dos dados, foi utilizada a plataforma de salto jump system optical 1.0, da marca cefise. Para a aplicação da BNM no grupo CB foi utilizado a bandagem neuromuscular da marca Teradex Tape.

Para a realização dos saltos, os voluntários foram instruídos pelo pesquisador a saltarem da seguinte forma:

Na posição inicial (Figura 3) o indivíduo realizou uma flexão de $110^{\circ}$ do joelho, leve flexão de tornozelo e mãos sobre o quadril para impedir movimento de membros superiores e sem inclinar excessivamente o tronco para frente.

Durante a fase de voo (figura 4) o indivíduo foi orientado a permanecer com o joelho em extensão, com as mãos apoiadas sobre o quadril.

$\mathrm{Na}$ fase de aterrisagem (Figura 5) o indivíduo deveria realizar uma leve flexão de joelho e realizar o salto de forma contínua, até a exaustão. 


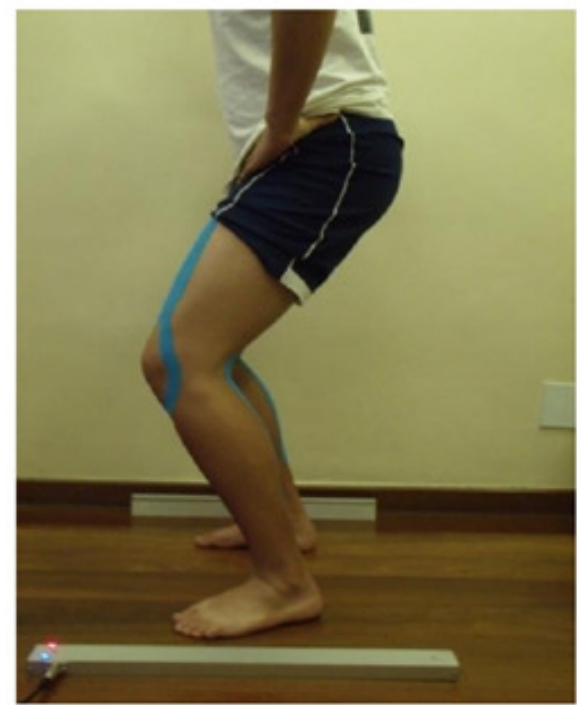

Figura 3: Fase inicial. Flexão de joelho a $110^{\circ}$, com as mãos sobre o quadril.

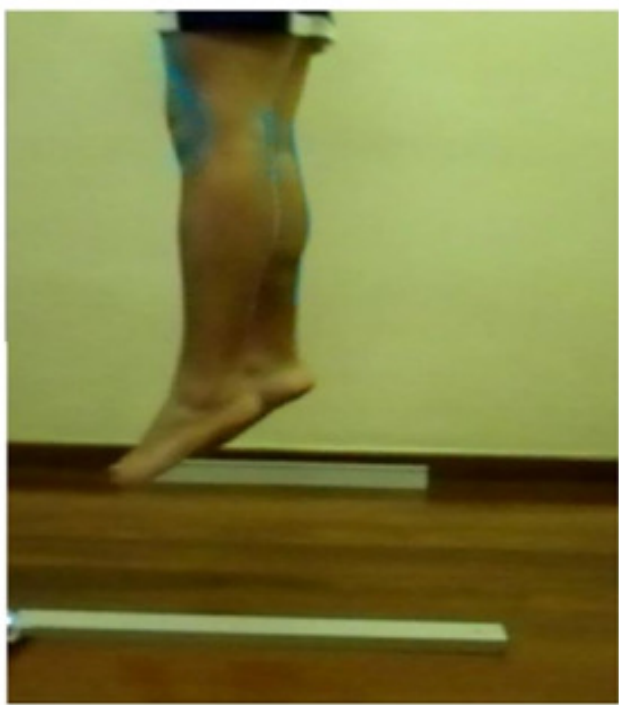

Figura 4: Fase de vôo. O indivíduo deveria permanecer no ar com extensão de joelhos.

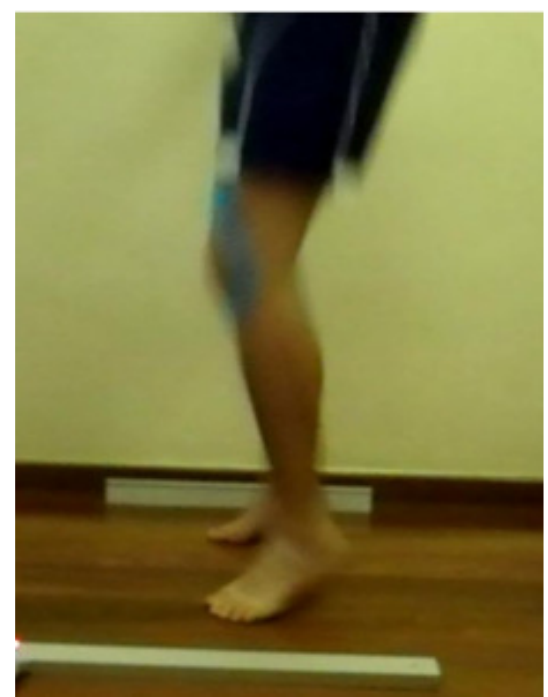

Figura 5: Fase de aterrisagem. Realizar saltos contínuos.

Depois de instruídos, os voluntários saltaram cinco vezes para conscientização da realização ideal dos saltos. Os voluntários foram orientados a realizar o salto com o pé diretamente em contato com o solo, ou seja, sem calçados ou meias. A participação dos candidatos em cada grupo foi realizada em um total de duas semanas. No grupo CB o indivíduo executava uma série de saltos no momento pré e no momento pós 10 minutos a aplicação da BNM. No $2^{\circ}$ dia (pós 24 horas) foi realizada apenas uma série de saltos, assim como no $3^{\circ}$ dia (pós 48 horas). Em seguida, o voluntário teve descanso de cinco dias e após esse tempo iniciou sua participação no outro grupo. No grupo CNTRL foi realizado o mesmo procedimento, apenas não foi aplicado a BNM.

Os dados captados pela plataforma de salto foram analisados através do programa jump system 1.0, disponível no site: http://cefise.com.br/, que fornecia as variáveis (número de saltos, tempo de voo, tempo de solo, altura, potência absoluta e potência relativa) necessárias para a avaliação estatística do estudo. A fim de analisar a normalidade da amostra, os dados foram submetidos ao teste de D'agostino. Em seguida foi realizado o teste ANOVA a fim de identificar se existia alguma diferença 
estatisticamente significativa, considerada quando $\mathrm{p}<0,05$.

\section{RESULTADOS}

A fim de demonstrar a caracterização da amostra, foram analisados os valores antropométricos dos voluntários colhidos no primeiro dia da coleta de dados, sendo expostos em média e desvio padrão (Tabela 1).

Tabela 1: Dados antropométricos. DP= Desvio Padrão

\begin{tabular}{cccc}
\hline & Idade & Altura & Massa \\
\hline Média & 22 & 1,73 & 80,08 \\
\hline DP & 2 & 0,05 & 13,4 \\
\hline
\end{tabular}

Ao analisar o número de saltos dos indivíduos constatou-se que não houve diferença estatisticamente significativa $(p>0,05)$ entre os grupos com bandagem $(C B)$ e controle (CNTRL) não ocorrendo interferência na resistência à fadiga. (Figura 6).
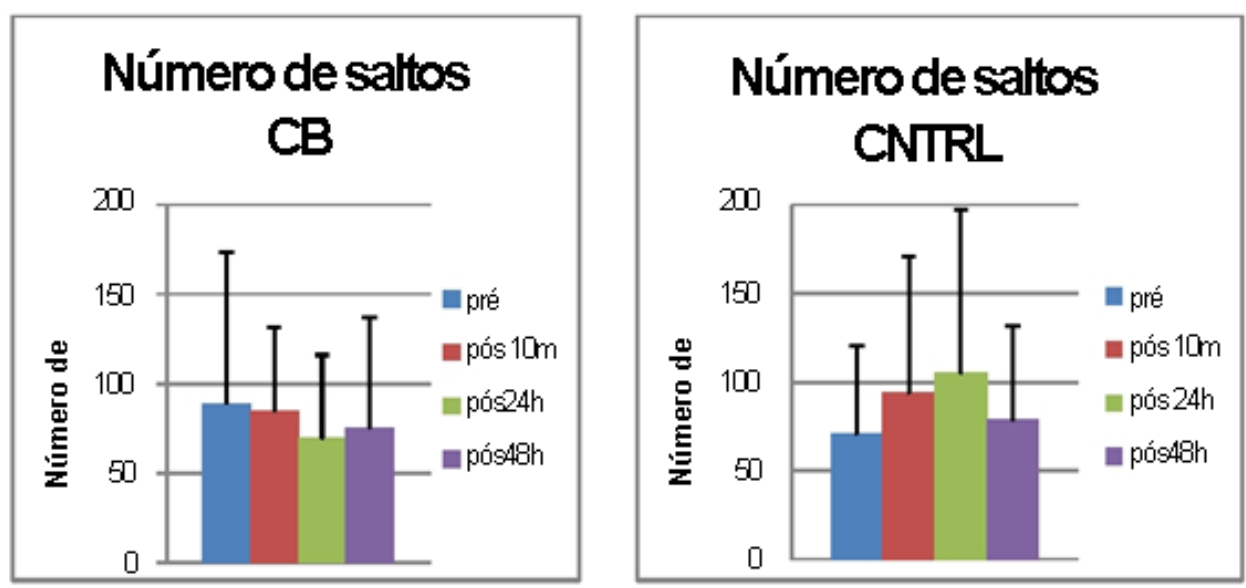

Figura 6: Número de saltos. Gráfico representando o número de saltos realizados pelo grupo $\mathrm{CB}$ e CNTRL, não havendo diferença estatística ( $p>0,05)$, o que indica que o uso da BNM não alterou a resistência à fadiga.

Os valores foram analisados através da média e desvio padrão em ambos os grupos. Quando analisado o tempo de solo observou-se que não houve diferença estatisticamente significativa ( $\mathrm{p}>0,05)$ entre os grupos com bandagem (CB) e controle (CTRL), o que mostra que não ocorreu variação no impulso do salto. (Figura 7). 

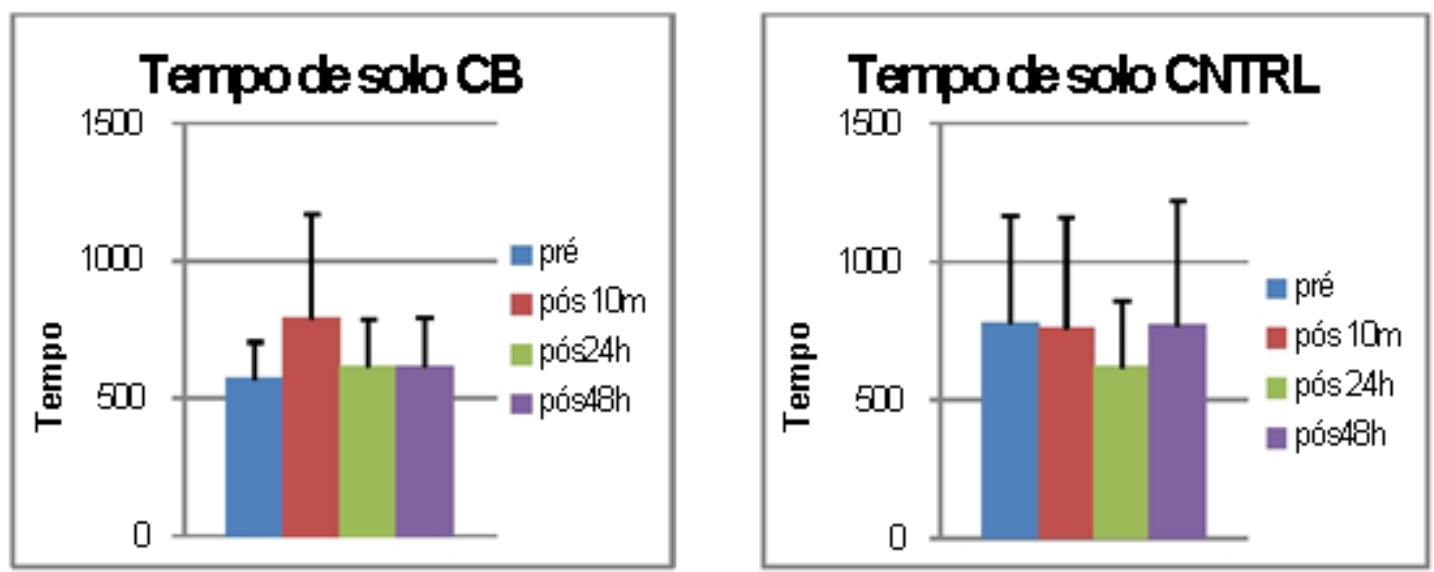

Figura 7: Tempo de solo. Gráfico que representa o tempo (ms) em que os voluntários realizaram o impulso entre os saltos, não apresentando diferença estatística entre os grupos CB e CNTRL $(\mathrm{p}>0,05)$.

A análise do tempo de voo foi realizada através da média e desvio padrão. No grupo CNTRL foi observado 506,6 + 152,3ms; $449+50,1 \mathrm{~ms} ; 492,8+106,3 \mathrm{~ms} ; 465+$ $50,2 \mathrm{~ms}$ para os momentos pré, pós $10 \mathrm{~min}$, pós $24 \mathrm{~h}$ e pós $48 \mathrm{~h}$, respectivamente. No grupo CB observou-se $467,9+37,1 \mathrm{~ms}$ no momento pré; $451,3+58,8 \mathrm{~ms}$ no pós $10 \mathrm{~min}$; $446+31,4 \mathrm{~ms}$ no pós $24 \mathrm{~h}$; e $460,5+56,8$ no pós $48 \mathrm{~h}$. Verificou-se que não houve diferença estatisticamente significativa $(p>0,05)$ entre o grupo com bandagem $(C B)$ e o grupo controle (CTRL) o que indica que não houve aumento da força gerada pelos membros inferiores no grupo CB. (Figura 8).
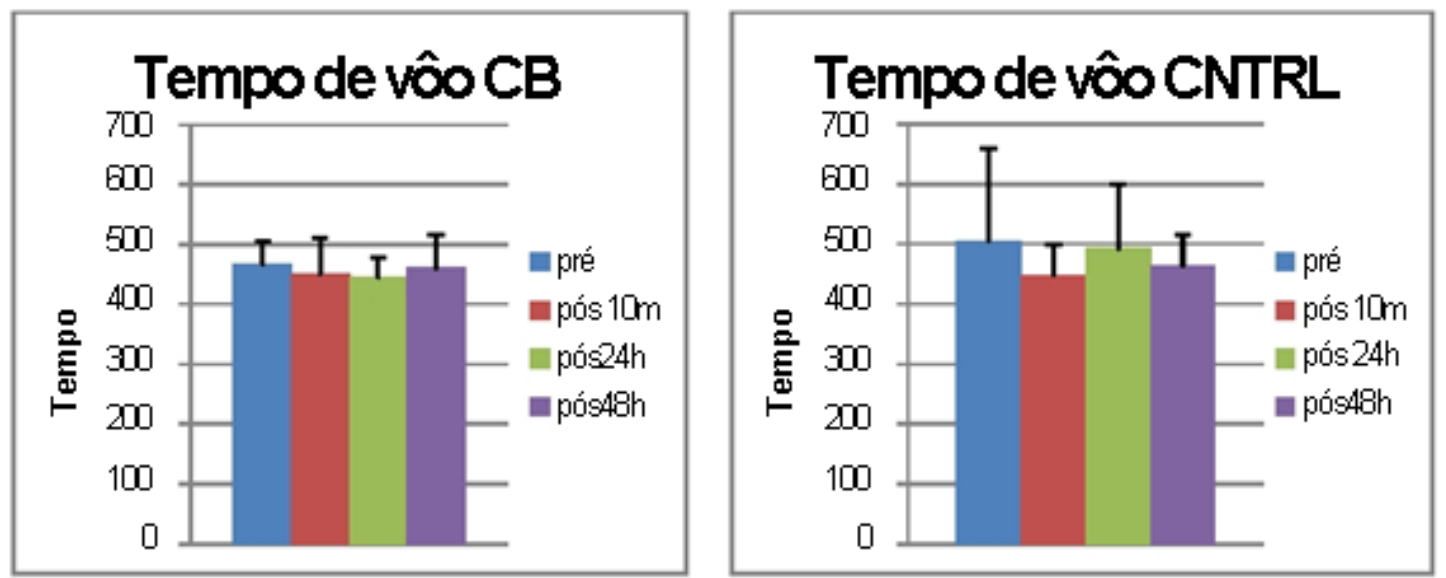

Figura 8: Tempo de voo. Demonstra o tempo que o indivíduo permaneceu no ar, relacionado com a com a força de impulso realizada. Não se observou alteração entre os grupos CB e CNTRL $(\mathrm{p}>0,05)$.

Analisando a altura dos saltos verificou-se que não houve diferença estatisticamente significativa $(p>0,05)$ entre o grupo com bandagem (CB) e o grupo controle (CTRL) indicando que não ocorreu alteração na força dos voluntários. (Figura 9). No grupo CB teve média de altura de $26,7+4,3 \mathrm{~cm}$ no momento pré; $27,6+6,4 \mathrm{~cm}$ no momento pós 10 min; $24,5+3,3 \mathrm{~cm}$ no pós $24 \mathrm{~h}$; e $26,3+6,4 \mathrm{~cm}$ no pós $48 \mathrm{~h}$. No grupo CNTRL apresentaram-se nos momentos pré, pós $10 \mathrm{~min}$, pós $24 \mathrm{~h}$ e pós $48 \mathrm{~h}$, os valores: 26,7 $+4,8 \mathrm{~cm} ; 24,9+5,5 \mathrm{~cm} ; 26,5+5,9 \mathrm{~cm} ; 26,8+5,1 \mathrm{~cm}$, respectivamente. 

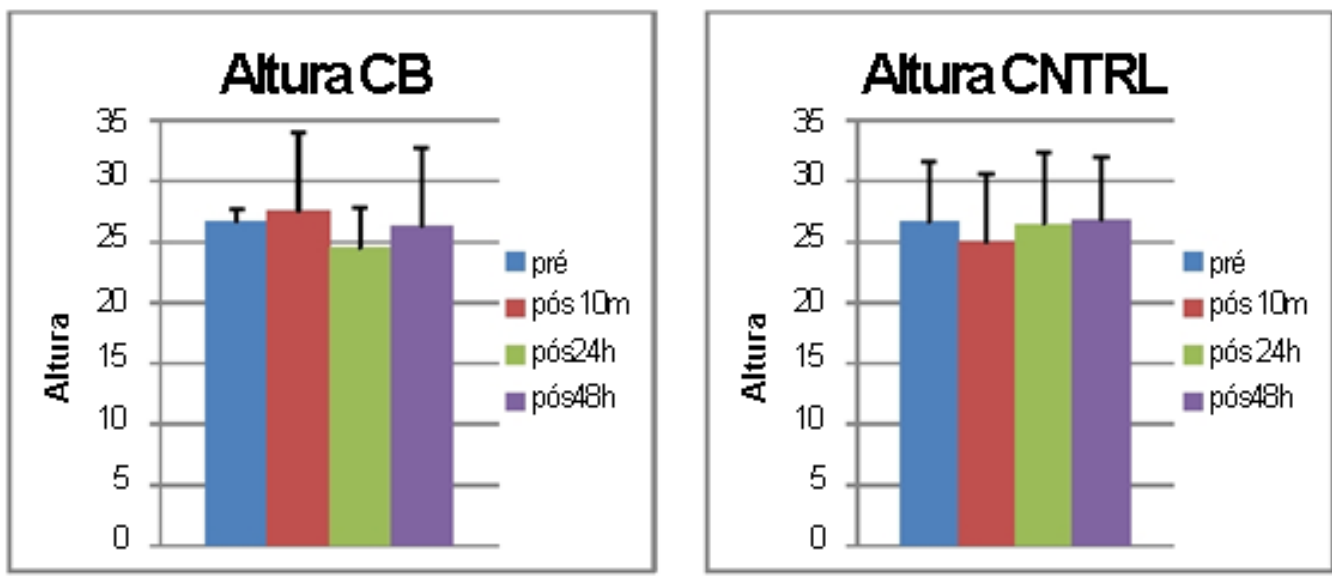

Figura 9: Altura. Apresenta descrição gráfica da altura (em forma de média + desvio padrão) dos saltos dos grupos CB e CNTRL, indicando que não houve qualquer diferença significativa $(\mathrm{p}>0,05)$.

De acordo com a análise da potência relativa ( $\backslash \mathrm{Kg})$, constatou-se que não houve diferença estatisticamente significativa $(p>0,05)$ entre o grupo com bandagem $(C B)$ e $o$ grupo controle (CTRL), ou seja, a aplicação de BNM não foi suficiente para promover melhora da potência relativa (levando em consideração a massa corporal). (Figura 10). O grupo CNTRL apresentou valores de 39,9 + 3,8 no momento pré; $39+3,4$ no pós 10 min; $39,7+4,2$ no pós $24 \mathrm{~h}$; e 40,8 + 3,7 no pós $48 \mathrm{~h}$. No grupo CB obteve 40,3 + 2,9; $40,6+4,5 ; 38,6+2$; e 39,8 +4,3, para os momentos pré, pós 10 min, pós 24 h e pós $48 \mathrm{~h}$, respectivamente.
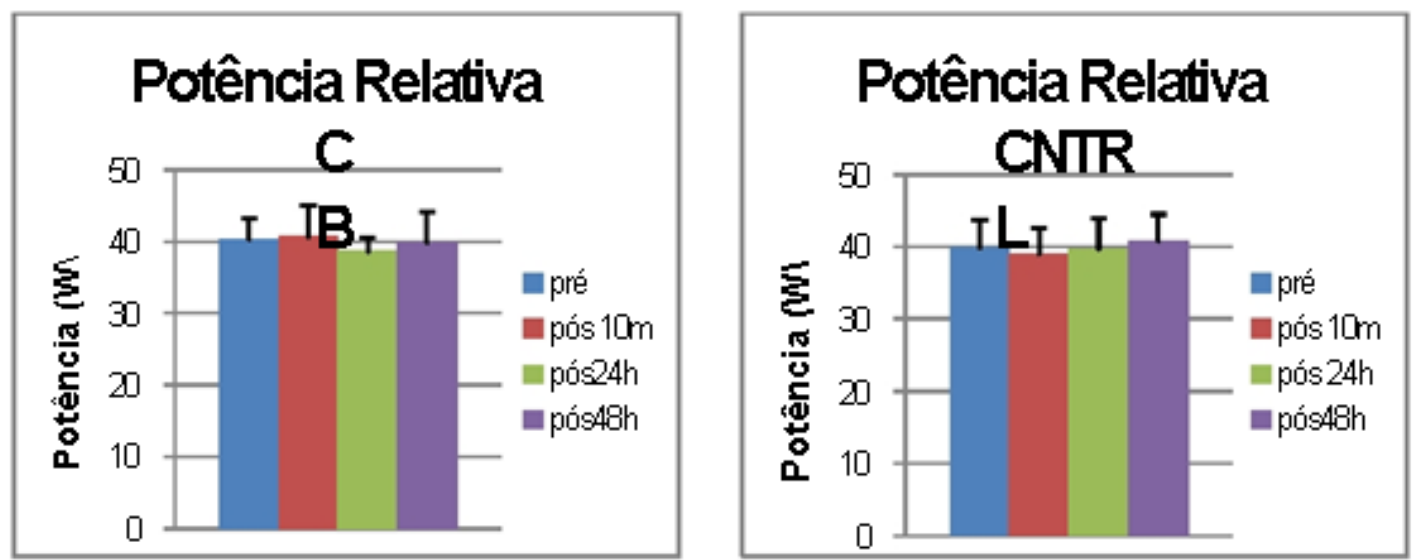

Figura 10: Potência Relativa.Representação gráfica da potência relativa (média + desvio padrão) gerada nos grupos CB e CNTRL, não havendo diferença estatística $(p>0,05)$ em nenhum momento.

$\mathrm{Na}$ análise da potência absoluta observou-se que não houve diferença estatisticamente significativa ( $>0,05)$ entre o grupo com bandagem $(\mathrm{CB})$ e o grupo controle (CTRL). (Figura 11). 

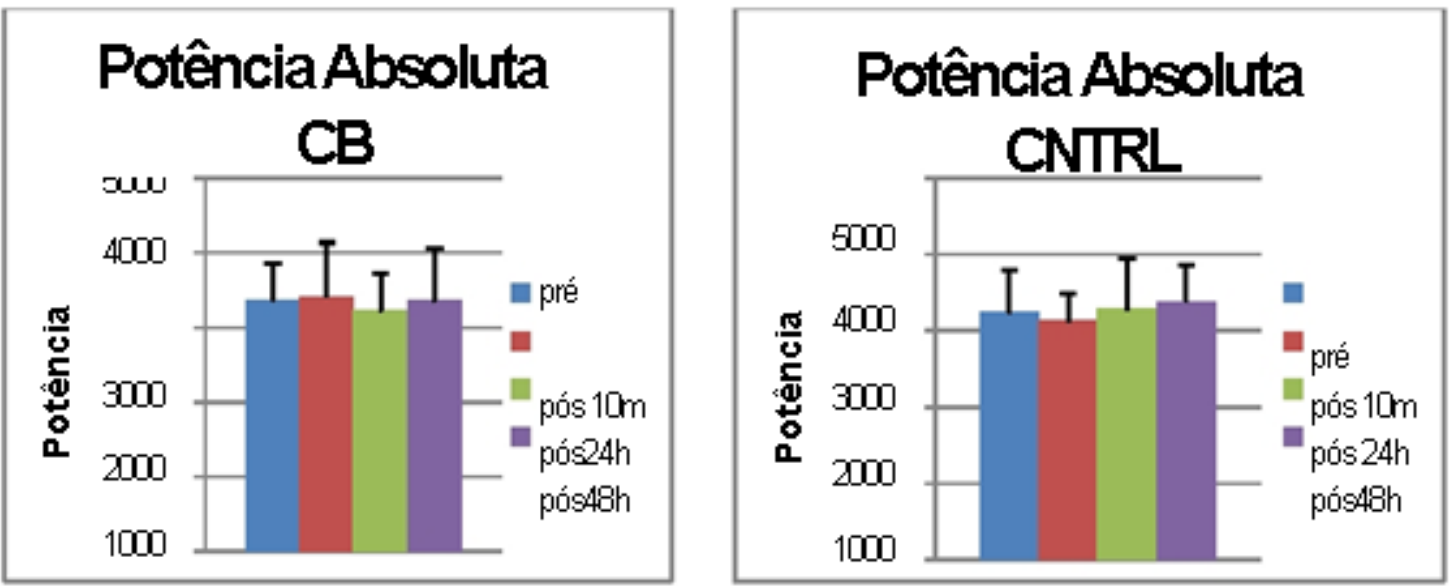

Figura 11: Potência Absoluta. Representação gráfica da potência absoluta produzida pelos grupos

CB e CNTRL, indicando que o uso da BNM não interferiu na potência absoluta de forma significativa $(\mathrm{p}>0,05)$.

\section{DISCUSSÃO}

A BNM ganhou aceitação mundial graças ao esporte, principalmente após os jogos olímpicos, realizado em Pequim, 2008. De acordo com seu criador, Kenso Kase, a BNM assume, dentro de outras possibilidades, a função de auxiliar a contração muscular, regulando seu tônus (KASE et al, 2003). A mídia, principalmente a esportiva, divulga a BNM como uma técnica terapêutica capaz de influenciar diretamente na performance e no rendimento do atleta em diversas modalidades esportivas, porém esta divulgação não apresenta embasamentos em evidências científicas que comprovem seu efeito sobre o desempenho. Com isto, o presente estudo comprometeu-se em averiguar se o uso da BNM influenciaria no desempenho dos indivíduos na realização do salto vertical.

De acordo com Hall (2005), o músculo possui a capacidade de responder a um estímulo mecânico externo através do desenvolvimento de tensão. A partir disto, acredita-se que a força elástica da BNM ao ser aplicado sobre a pele estimula mecanoceptores presentes através da sua retração elástica, levando o músculo a gerar tensão de acordo com o sentido da retração (KASE et al, 2003).

Estudos recentes com análise eletromiográfica comprovam que a BNM incrementa o recrutamento de unidades motoras, porém há divergências quanto ao tempo necessário para a mesma apresentar um efeito significativo. Em seu estudo, Slupik et al (2007) procurou verificar se a BNM altera o recrutamento do músculo vasto medial durante contrações isométricas em 27 pessoas saudáveis e identificou que a BNM otimiza de forma significativa o recrutamento das unidades motoras do músculo. Segundo seus resultados, a BNM foi capaz de promover o incremento da atividade eletromiográfica do vasto medial após 24 horas da sua aplicação e se mantém até 48 horas após a retirada da mesma. Mais recentemente, Rodrigues e de Souza (2009) concluíram em seu estudo que a aplicação da BNM com a técnica de ativação do músculo deltoide resultou no aumento do recrutamento muscular somente após 48 horas do momento da aplicação.

Halseth et al (2004) buscou determinar a influência da BNM na propriocepção do tornozelo comparando grupos com e sem a BNM. De acordo com seus resultados, concluiu-se que a BNM não altera na propriocepção do tornozelo, porém vale destacar 
que o estudo analisou a resposta proprioceptiva nos movimentos de flexão e inversão separadamente e, segundo Magee (2005), a entorse de tornozelo por inversão ocorre com o pé em inversão, adução e flexão de forma simultânea. Por este motivo, Barreto et al (2010) analisaram o efeito proprioceptivo da BNM na entorse de tornozelo simulando a entorse por inversão, confeccionando uma plataforma capaz de simular fidedignamente o movimento de flexão e inversão. Os resultados revelaram que a utilização da BNM sobre os músculos fibulares apresentou diferença significativa no tempo de resposta do músculo e, consequentemente, na melhora da propriocepção.

Com esses achados, somados a popularização da técnica vem ganhando espaço uma nova aplicação no esporte, que propõe melhora na performance muscular e desportiva, dentro desse contexto buscou-se verificar se o uso das técnicas de ativação são capazes de promover incremento no desempenho em saltos verticais.

Para Hespanhol e de Arruda (2008) a eficiência do indivíduo no salto vertical está não só relacionada ao sistema de recrutamento e sincronização muscular, mas também contribuição do componente contrátil e elástico. Hall (2005) conceitua componente contrátil como uma capacidade muscular em permitir o desenvolvimento de tensão através da estimulação de fibras musculares. Ainda afirma que o componente elástico pode ser dividido de duas formas: o componente elástico em paralelo (responsável em fornecer resistência a um estiramento muscular passivo) e o componente elástico em série (localiza-se nos tendões e é capaz de armazenar energia elástica quando um músculo é estirado, produzindo um recuo elástico que possivelmente aumenta a produção de força).

Desta forma, o presente estudo identificou que o uso da BNM não foi capaz de promover aumento no desempenho do salto vertical (analisando o tempo no solo, tempo de voo, altura, potência absoluta e potência relativa) nem a resistência a fadiga (analisando o número total de saltos). Apesar de estudos demonstrarem que a BNM otimiza o recrutamento de unidades motoras (SLUPIK et al, 2007; BARRETO et al, 2010; a RODRIGUES e DE SOUZA, 2009), isto não quer dizer que a performance do salto vertical também será alterada, pois neste movimento funcional ocorre uma contração excêntrica seguida imediatamente de uma contração concêntrica (ciclo de alongamento- encurtamento), tendo como principal fator o componente elástico em série (HALL, 2005), componente este que provavelmente a bandagem não consiga promover alteração, pois é possível que a BNM possa estimular o componente elástico em paralelo, aumentando a resistência do músculo em realizar um alongamento, alterando na velocidade do ciclo de alongamento-encurtamento e, consequentemente, na utilização do componente elástico em série.

A fim de averiguar o desempenho do salto vertical com o uso da BNM foi analisada a altura, o tempo de voo, o tempo no solo, a potência absoluta (dada em Watts) e a potência relativa (dada em Watts/Kg), não havendo nenhuma diferença estatística ( $\mathrm{p}>0,05)$ entre o grupo CNTRL e o grupo CB, ou nos momentos CB pré e CB pós $10 \mathrm{~min}$, pós 24 horas e pós 48 horas. Também não foi encontrada nenhuma diferença significativa $(\mathrm{p}>0,05)$ na resistência a fadiga, ao analisar o número de saltos realizados no grupo CNTRL e grupo CB.

Os resultados deste estudo parecem concordar com Huang et al (2011), que ao analisar a performance (altura) e a atividade eletromiográfica do músculo tríceps sural durante o salto vertical com o uso da BNM constatou que apesar de aumentar o

Persp. online: biol. \& saúde, Campos dos Goytacazes, 7 (2), 62-75, 2012 seer.perspectivasonline.com.br 
recrutamento muscular, não houve uma diferença significativa na altura do salto vertical entre os indivíduos do grupo com a aplicação da BNM e do grupo placebo. Fu et al (2008) também concluiu de acordo com seus resultados que a BNM não é capaz de intervir na capacidade de gerar força muscular em indivíduos saudáveis ao avaliar, através de um aparelho isocinético, a força muscular dos músculos quadríceps e isquiotibiais após aplicação imediata e após 12 horas de aplicação da BNM.

Tendo em vista os resultados expostos, este estudo sustenta a hipótese de que a BNM não pode ter como função a melhora da performance em indivíduos saudáveis, uma vez que sua aplicação não influenciou no desempenho do salto vertical realizados de forma contínua. Também é sustentado por estudos que se propuseram a avaliar o efeito da BNM no desempenho (HUANG et al, 2011) e na capacidade de gerar força muscular (FU et al, 2008).

De acordo com evidências científicas encontradas na literatura, a BNM se apresenta como um recurso terapêutico importante no processo de reabilitação, porém ainda não possui respaldo científico quanto a sua capacidade de influenciar na performance do indivíduo. Estudos comprovam que a BNM é eficaz em diversas condições clínicas, como: otimizar a propriocepção do tornozelo na entorse por inversão (BARRETO et al, 2010); diminuir da subluxação de ombro em indivíduos com Acidente Vascular Cerebral (SANTOS et al, 2010); melhorar a função e controle muscular na reabilitação pediátrica (YASUKAWA et al, 2006); aumentar a atividade elétrica muscular (SLUPIK et al, 2007; RODRIGUES e de SOUZA; 2009); auxiliar no tratamento de disfunções posturais, como a escoliose (BAC et al, 2009); contribuir no processo de reabilitação com regressão de sintomas dolorosos (THELEN, DAUBER, STONEMAN, 2008; CLELAND et al, 2009; REICH, 2009).

\section{CONCLUSÃO}

De acordo com os resultados obtidos neste estudo, pode-se concluir que o uso da BNM não produz alterações neurofisiológicas suficientes capazes de influenciar o desempenho de indivíduos saudáveis e sedentários na execução do salto vertical ou na capacidade do organismo de retardar os efeitos da fadiga muscular. A dificuldade de padronizar a execução dos saltos verticais foi identificada como um fator limitante deste estudo. Mesmo com orientação prévia a execução dos saltos a fim de conscientizar os voluntários, foi constatada uma variação na forma de realização do salto vertical, identificado pela heterogeneidade da variável tempo no solo, alterando o tempo necessário para o indivíduo realizar o impulso contra o chão. Esta dificuldade parece estar relacionada com a população escolhida, assim como o tipo de salto avaliado. Desta forma, faz-se necessário investigações do efeito da BNM no desempenho do salto vertical em atletas, principalmente aqueles que realizam o salto vertical constantemente, e a escolha de outras formas de salto.

\section{REFERÊNCIAS}

BAC A.et al; Efficacy of Kinesiology Taping in the rehabilitation of children with lowangle scoliosis. Medsportpress, V.9, N. 3(4); 2009.

BARRETO R, AZEVEDO R. Efeito da Bandagem Neuromuscular em atletas de futebol durante a simulação entorse de tornozelo por inversão: uma análise eletromiográfica. Revista Perspectivas online, V.4, No 13, pp.181-193; 2010. 
CLELAND J.; Short-Therm effects of cervical kinesio taping on pain and cervical range of motion in patients with acute whiplash injury: a randomized; Journal of orthopaedic and sports physical therapy, v. 39, n. 7, 2009.

FU, T.; Effect of kinesio taping on muscle strength in athletes- a pilot study; Journal of Science and Medicine in Sport, V. 11, pp. 198-201, 2008.

FURTADO G. de S.; de MELO R. R.; GARCIA M. A. C; Desempenho de atletas de voleibol do sexo feminino em saltos; Revista eletrônica da Escola de Educação Física e Desportos- UFRJ; V.2, N.2; 2006

GARCÍA-LÓPEZ, J. et al; El tipo de plataforma de contacto influye en El registro de La altura de salto vertical estimada a partir Del tiempo de vuelo Motricidad. European Journal of Human Movement, V.21;pp. 1-24, 2008.

HALL, S. BiomecânicaBásica, $4^{\mathrm{a}}$ edição, RJ, Editora Guanabara, 2005

HALSETH T. et al. The effects of kinesio taping on proprioception at the ankle Journal of Sports Science and Medicine;V3, 1-7, 2004.

HESPANHOL, J. E. ; de ARRUDA, M.; Saltos Verticais, $1^{\text {a }}$ Edição. São Paulo, Phorte Editora, 2008.

HUANG, C. et al; Effect of de kinesio tape to muscle activity and vertical jump performance in healthy in active people. Biomedical Engineering OnLine, vol. 10, 70; 2011.

KASE K., WALLIS J., KASE T.,Clinical Therapeutic Applications of the Kinesio Taping Method. Tokyo, Japan: Ken IkaiCoLtd; 2003.

MAGEE, D. J. Avaliação Musculoesquelética, 5a Edição. São Paulo, Editora Manole, 2010.

PEREIRA R. et al; Jump test: comparação da performance pelo método clássico e através do foot swich. Fitness and performance journal, V. 8, N..2, pp.73-78, 2009.

REICH, B.; Kinesiotape as an adjunct to traditional physical therapy in the treatment of a patient with chronic low back pain: a case report, Tese (Doutorado em Fisioterapia - Carroll University.), 2009.

RODRIGUES, R. N., de SOUZA, L. S.; Efeitos da bandagem neuromuscular sobre o recrutamento do músculo deltoide. Monografia (Curso de fisioterapia ISECENSA), 2009.

SANTOS, J. C. C.; GIORGETTI, M. J. S.; TORELLO, M; MENEGHETTI C. H. Z.; ORDENES, I. E. U. A influência da KinesioTaping no tratamento da subluxação de ombro no acidente vascular cerebral. Revista de neurociência, 2010. 
SIJMONSMA, J. Taping Neuro Muscular Manual. $1^{\text {a }}$ d. Portugal: Cascais. p. 13-18, 2007.

SILVA K.R; MAGALHÃES J; GARCIA M.A.C. Desempenho do salto vertical sob diferentes condições de execução. Arquivos em Movimento, Rio de Janeiro, v.1, n.1, p.17-24;2008:

SLUPIK A. et al., Effect of Kinesio Taping on bioelectrical activity of vastus medialis muscle. Medsportpress, 6(6); Vol. 9, 2007.

THELEN M., DAUBER J, STONEMAN P. The clinical efficacy of kinesio tape for shoulder pain: A randomized, Double-blinded, clinical trial.

Journal oforthopaedic sports physicaltherapy. V 38, n 7, 2008

YASUKAWA, A., PATEL, P., \&SISUNG, C. Pilot study: Investigating the effects of Kinesio Taping ${ }^{\circledR}$ in an acute pediatric rehabilitation setting. American Journal of Occupational Therapy, 60, 104-110, 2006. 